\title{
The Inoue balloon for dilatation of the tricuspid valve: a modified over-the-wire approach
}

\author{
T R D Shaw
}

\begin{abstract}
The Inoue balloon was used for dilatation of tricuspid stenosis in a 74 year old woman. The valve was reached by an over-the-wire approach with a 0.025 exchange length guide wire. The Inoue stylet would not reach the tricuspid orifice because the right atrium was so large. The Inoue balloon's special dilatation characteristics allowed good positioning at the tricuspid orifice. After dilatation to $27.5 \mathrm{~mm}$, the pressure drop across the valve was reduced from 12 to $5 \mathrm{~mm} \mathrm{Hg}$. Further dilatation at $30 \mathrm{~mm}$, however, created moderately severe tricuspid reflux without a further reduction of gradient.

The Inoue balloon is suitable for dilatation of tricuspid stenosis but small increments in dilatation size may be required for optimal reduction in gradient without creating significant reflux.
\end{abstract}

There is still only limited experience of balloon dilatation for tricuspid stenosis but there have been reports of successful relief of tricuspid stenosis, rheumatic ${ }^{1-4}$ and congenital ${ }^{5}$ and of combined dilatation of the tricuspid and mitral valves $^{67}$ and even triple valve dilatation of tricuspid, mitral, and aortic stenosis. ${ }^{8}$ Tricuspid bioprosthetic valves have also been improved by balloon dilatation. ${ }^{910}$ In these cases, standard tubular polyethylene or polyvinyl balloons have been used, being passed to the tricuspid valve orifice over a guide wire advanced to the pulmonary artery or right ventricular cavity. In the patient reported here, it was decided to use the Inoue balloon, though it was designed for the mitral valve, because the patient had a giant right atrium and a very small right ventricle-features which would have made placement of a tubular balloon at the orifice much more difficult. The design of the Inoue balloon is unique. Its walls contain a network of synthetic nylon fibres which give different compliance characteristics to its distal, proximal, and middle segments, so that at inflation the distal portion of the balloon enlarges first, allowing this portion to be drawn back against the stenosed orifice. On further inflation, the proximal segment enlarges, holding the balloon at the vale orifice which is then dilated as the middle segment expands at full inflation. The short length of the Inoue balloon and its blunt distal point reduce the risk of perforating the myocardial wall. The deflated Inoue balloon does not have a low profile, and to allow passage through the skin (and though the atrial septum for dilatation of the mitral valve) a metal stretching tube is included in the balloon equipment set. This is inserted into the balloon catheter lumen and causes the deflated balloon to be stretched into a lower, more rigid shape. The Inoue balloon catheter has no intrinsic curve and when it is used to dilate the mitral valve a curved stylet wire is inserted to arc the balloon towards the mitral valve. When the Inoue balloon was used to dilate the tricuspid valve in this case, it was found possible to use it in a modified over-the-wire approach.

\section{Case report}

The patient was a 74 year old women who had no history of rheumatic fever. However, mitral valve disease had been noted during her first pregnancy at age 22 . When aged 61 , she had a pacemaker implanted because of lightheadedness caused by episodes of sinus arrest. The surgeon was unable to pass the pacing electrode across the tricuspid valve and an epicardial pacing system was implanted. A diagnosis of mild mitral stenosis, mild mitral reflux, and moderate tricuspid stenosis was made at this time. When she was 67 the pacemaker ceased functioning but she was then in atrial fibrillation without excessively long RR intervals and the pacemaker was not renewed. Marked ascites was noted at age 70 ; this responded to diuretic treatment. By the age of 74 she had breathlessness at rest and was restricted to walking 50 yards. She now had chronic ascites despite taking frusemide $160 \mathrm{mg}$ per day. Echocardiography showed she still had mild mitral stenosis (pressure half time $125 \mathrm{~ms}$ ), mild mitral reflux, and severe tricuspid stenosis (pressure half time $300 \mathrm{~ms}$ : estimated valve area $0.7 \mathrm{~cm}^{2}$ ). She had chronic anaemia due to diverticulitis and telangiectasia of the stomach. She had had a previous nephrectomy and had moderate renal impairment. She was referred to the Western General Hospital for balloon dilatation of the tricuspid valve.

On examination, she was a thin, frail woman $(45 \mathrm{~kg}$, height $159 \mathrm{~cm}$ ) in atrial fibrillation with a controlled ventricular rate. The jugular venous pressure was raised to beyond the ears and the liver was four finger breadths enlarged and slightly pulsatile. She had moderate ascites 


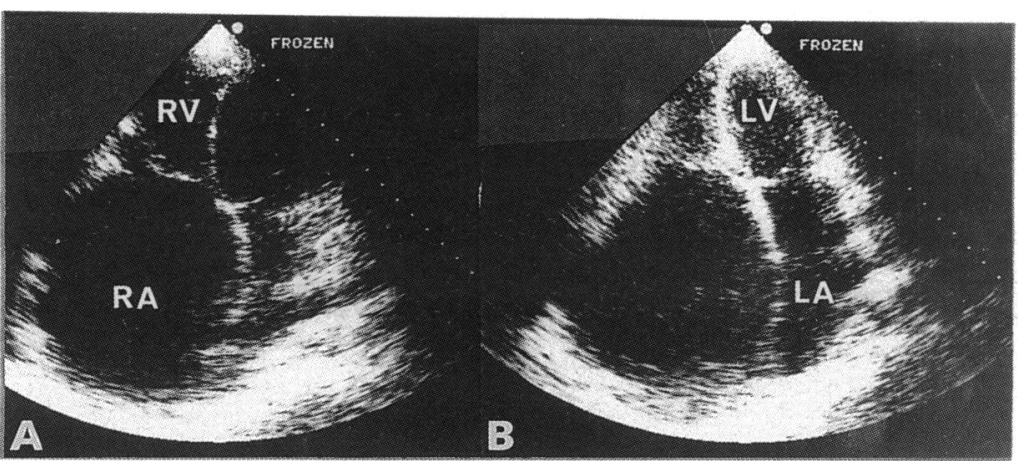

Figure 1 Apical four chamber views to show right heart $(A)$ and left heart $(B)$.

with slight sacral and ankle oedema. On auscultation a 3/6 tricuspid diastolic murmur and $1 / 6$ mitral diastolic murmur were audible.

At echocardiography (fig 1) the mitral valve was only mildly stenosed with slight cusp thickening. Left ventricular function was normal. The right atrium was huge $(9.7 \times 10.8 \mathrm{~cm}$ in the apical view) and the atrial septum bulged into the left atrial cavity. The tricuspid valve cusps were thin but showed minimal opening.

At right heart catheterisation, the $3 \mathrm{~cm}$ curve Brockenbrough catheter and 0.035 exchange guide wire were negotiated with difficulty through the tricuspid valve. Right atrial pressure was $33 / 22$ (mean 26) $\mathrm{mm} \mathrm{Hg}$ and right ventricular pressure was $47 / 5-16 \mathrm{~mm} \mathrm{Hg}$. Cardiac output was $3.7 \mathrm{1} / \mathrm{min}$ and diastolic filling time $37 \mathrm{~s} / \mathrm{min}$ with a mean tricuspid transvalvar gradient of $12 \mathrm{~mm} \mathrm{Hg}$, giving a valve area,

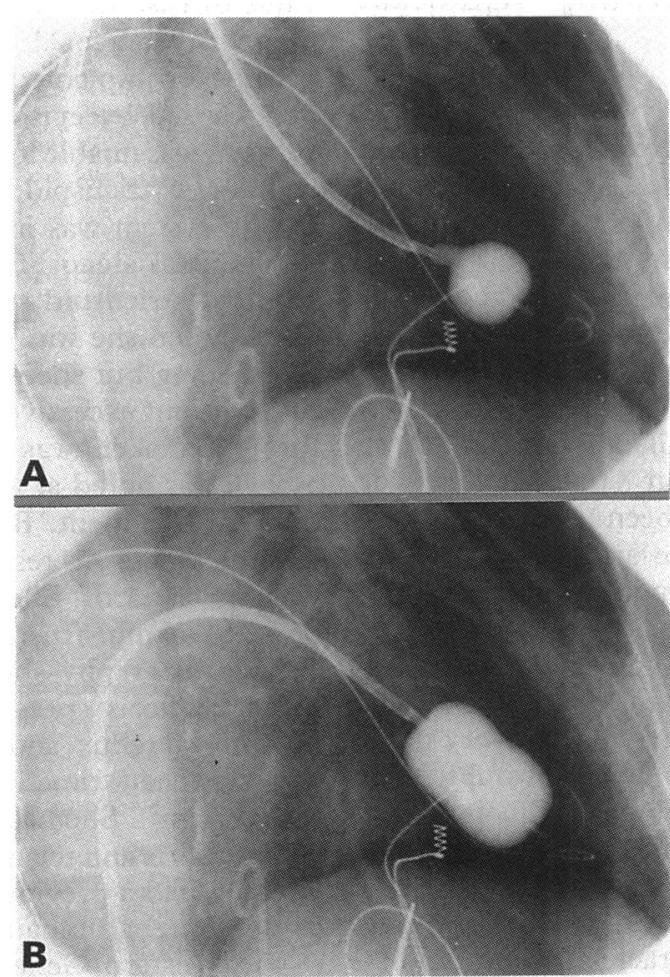

Figure 2 Inoue balloon dilatation at the tricuspid valve (A) The distal portion of the balloon has been dilated. (B) The balloon catheter has been pulled back to engage (B) The balloon catheter has been pulled back to engage
the distal portion of the orifice and the balloon has been fully dilated. A 0.035 inch guide wire also lies across the tricuspid valve. An epicardial pacemaker system is in situ and an F6 pigtail catheter lies in the descending aorta to monitor systemic pressure. calculated from the Gorlin formula $(3700 \div$ $44.3 \times 37 \times \sqrt{12}$ ) of $0.65 \mathrm{~cm}^{2}$. A right ventricular injection of contrast confirmed the absence of tricuspid reflux.

The 0.035 inch exchange guide wire remained in the right ventricle, the Brockenbrough catheter was removed, and a Block double lumen catheter (USCI) was passed over the wire to the right ventricle. An exchange length 0.025 inch wire was then passed via the Block second lumen to lie alongside the 0.035 inch wire and the Block catheter was withdrawn. The Inoue balloon, with stretching metal tube inserted, was then passed percutaneously over the 0.025 inch wire to the right atrial cavity and the metal tube withdrawn. The 0.035 inch wire was retained within the right ventricle to maintain access across the tricuspid valve. The Inoue balloon was advanced over the 0.025 inch wire which took up a position around the superior border of the right atrium before descending to cross the tricupsid valve. The Inoue balloon followed over the wire and across the tricuspid orifice. The distal portion of the balloon was inflated and the Inoue catheter shaft was drawn back until the distal portion of the balloon was held against the tricuspid orifice. The balloon was then inflated to $27.5 \mathrm{~mm}$ (fig 2). On deflation, the Inoue balloon and 0.025 inch guide wire were ejected back into the right atrium. The Block double lumen catheter was re-advanced over the 0.035 inch wire to the right ventricle and its second lumen was used for pressure measurement simultaneously with right atrial pressure measurement via the Inoue balloon lumen. The tricuspid valve gradient had been reduced to $5 \mathrm{~mm} \mathrm{Hg}$ with no increase in valve reflux. It was decided to attempt a greater reduction of gradient. With its stylet inserted, the Inoue balloon pointed towards the tricuspid valve but would not reach to the orifice. The 0.025 inch guide wire was therefore replaced into the right ventricle through the double lumen catheter and the Inoue balloon again followed over the wire to the tricuspid orifice.

The valve was dilated to $30 \mathrm{~mm}$. A right ventricle angiogram now showed moderately severe tricuspid reflux: the valve gradient had incresed to $7 \mathrm{~mm} \mathrm{Hg}$, with right atrial pressure $32 / 18 \mathrm{~mm} \mathrm{Hg}$ and right ventricular pressure 39/5-18 mm Hg. Cardiac output had risen slightly to $3.9 \mathrm{l} / \mathrm{min}$ : assuming tricuspid reflux at $33 \%$ of stroke volume, valve area increased to $1.56 \mathrm{~cm}^{2}$.

The patient tolerated the procedure well and the next day she returned to the referring hospital and was discharged home a few days later. One month after the procedure her daughter reported that her breathlessness was much better and that the patient's general appearance and oedema were improved, with slight increase in exercise capacity. Doppler assessment showed that the tricuspid valve area had increased to $1.9 \mathrm{~cm}^{2}$. At seven months and one year after valve dilatation, there continued to be evidence of moderately severe tricuspid reflux and mild stenosis. The patient's condition then gradually declined and she died in heart failure 13 months after the procedure. 


\section{Discussion}

The Inoue balloon was used to dilate the tricuspid valve in this patient because of the considerable discrepancy between the atrial and ventricular sizes which would have made it difficult to obtain a stable orifice position with polyethylene balloons. Chen et al had previously used the Inoue balloon for combined dilatation of the tricuspid and pulmonary valves. ${ }^{11}$ The Inoue balloon, with its metal stretching tube inserted could be advanced over a 0.025 inch guide wire: the stretching tube was essential for passing the balloon percutaneously to reach the right atrial cavity. Once the stretching tube was removed the Inoue balloon followed by an over-the-wire approach to the tricuspid orifice. The Inoue stylet was unable to direct the balloon to the orifice with such a giant right atrium. This over-the-wire approach might also be applicable in occasional cases of mitral stenosis where there is difficulty in crossing the mitral orifice.

Considerable reductions in valve gradient and an increase in valve area have been reported for rheumatic tricuspid stenosis ${ }^{1-46-8}$ usually without development of tricuspid reflux. In the case report here, the final area was comparable to that obtained with double balloon dilatation but it was associated with the onset of significant tricuspid reflux. Bourdillon et al also found that reduction of stenosis was accompanied by the development of tricuspid incompetence: at subsequent tricuspid valve replacement, two of the three tricuspid commissures had been opened but no leaflet tear was found. ${ }^{12}$ To avoid tricuspid reflux, small progressive increases in dilatation diameter, as is possible with the Inoue balloon, may be an advantage in obtaining the optimal balance between gradient reduction and development of valve incompetence. In this patient we expected that it might be difficult to position the balloon and only two dilatation diameters were used: in retrospect, small increases might have given a better haemodynamic result, although in this valve dilatation could be carried out only at a very advanced stage of valve disease.

1 Al-Zaibag M, Ribeiro P, Al-Kasab S. Percutaneous balloon valvotomy in tricuspid stenosis. Br Heart $J$ 1987;57 $51-3$

2 Khalilullah M, Tyagi S, Yadav BS, Jain P, Choudhry A, Lochan $R$. Double-balloon valvuloplasty of tricuspid stenosis. Am Heart J 1987;114:1232-3.

3 Ribeiro P, Al-Zaibag M, Al-Kasab S, et al. Percutaneous double balloon valvotomy for rheumatic tricuspid stenosis. Am J Cardiol 1988;61:660-2

4 Goldenberg IF, Pedersen W, Olsen J, Madison JD, Mooney MR, Gobel FL. Am Heart J 1989;118:417-9.

5 Lokhandwala YY, Rajani RM, Dalvi BV, Kale PA. Successful balloon valvotomy in isolated congenital tricuspid stenosis. Cardiovasc Intervent Radiol 1990;13:354-6.

6 Bethencourt A, Medina A, Hermandez E, et al. Combined percutaneous balloon valvuloplasty of mitral and tricuspid valves. Am Heart J 1990;119:416-8.

7 Berland J, Rocha P, Mechmerche R, et al. Percutaneous valvuloplasty for combined mitral and tricuspid stenosis: valvulopiasty for combined mitral and tricuspid ste 3 cases. Arch Mal Coeur 1990;83:1585-9.

8 Konugres GS, Lau FYK, Ruiz CE. Successive percutaneous double-balloon mitral, aortic and tricuspid valvotomy in double-balloon mitral, aortic and tricuspid valvotomy in
rheumatic trivalvular stenoses. Am Heart J 1990;119: rheuma.

9 Wren C, Hunter S. Balloon dilatation of a stenosed bioprosthesis in the tricuspid valve position. Br Heart $J$ thesis in the

10 Chow W-H, Cheung K-L, Tai Y-T, Cheng C-H. Successful percutaneous balloon valvuloplasty of a stenotic tricuspid bioprosthesis. Am Heart J 1990;119:666-8.

11 Chen CR, Lo ZX, Huang ZD, Cheng TO. Concurrent percutaneous balloon valvuloplasty for combined tricuspid and pulmonic stenoses. Cathet Cardiovasc Diagn 1988;15:55-60.

12 Bourdillon PDV, Hookman LD, Morris SN, Waller BF. Percutaneous balloon valvuloplasty for tricuspid stenosis: haemodynamic and pathological findings. Am Heart $J$ 1989;117:492-5. 